\title{
Arthroscopic Capsular Release for the Stiff Shoulder
}

\section{Description of Technique and Anatomic Considerations*}

\author{
Robert M. Zanotti, MD, and John E. Kuhn, † MD
}

\section{From the Section of Orthopaedic Surgery, The University of Michigan, Ann Arbor, Michigan}

\begin{abstract}
The anatomic proximity of several neurovascular structures remains a major concern to the surgeon interested in performing arthroscopic capsular release. We evaluated the anatomic relationships between the released capsule and the axillary nerve, posterior circumflex humeral artery, and brachial artery in a frozen cadaveric model. With the aid of electrocautery, seven cadaveric shoulders underwent complete arthroscopic capsular release. The release was performed circumferentially, approximately $1 \mathrm{~cm}$ lateral to the glenoid rim. All shoulders were subsequently frozen and sectioned through the plane of the capsular release while the shoulder was maintained in the lateral arthroscopic position $\left(45^{\circ}\right.$ of abduction and $20^{\circ}$ of flexion). Anatomic dissection revealed an average distance from the capsular release to the axillary nerve of $7.04 \mathrm{~mm}$ (95\% confidence interval, $5.62,8.47$ ), to the posterior circumflex humeral artery of $8.2 \mathrm{~mm}$ (95\% confidence interval, $6.41,9.99)$, and to the brachial artery of $15.97 \mathrm{~mm}$ (95\% confidence interval, $9.85,22.09)$. As the axillary nerve was followed medially from the released capsule, the inferior border of the subscapularis muscle became interposed between the capsule and the axillary nerve. This limited anatomic study shows that a relatively safe margin between the capsule and the neighboring neurovascular structures can be obtained by releasing the capsule within $1 \mathrm{~cm}$ of the glenoid rim.
\end{abstract}

\footnotetext{
* Presented at the 22nd annual meeting of the AOSSM, Lake Buena Vista, Florida, June 1996.

† Address correspondence and reprint requests to John E. Kuhn, MD, The University of Michigan Shoulder Group, 24 Frank Lloyd Wright Drive, PO Box 363, Ann Arbor, Michigan 48106-0363.

No author or related institution has received any financial benefit from research in this study.
}

Glenohumeral capsular contractures can be a significant cause of disabling shoulder pain and can limit function. These contractures can occur without apparent cause, as in adhesive capsulitis, may be iatrogenic, as in the case of limited motion after operative procedures for anterior instability of the shoulder, or may be associated with other disorders of the glenohumeral joint, such as osteoarthritis. The initial management of adhesive capsulitis has consisted of supportive treatment followed by medications such as nonsteroidal antiinflammatory agents, supervised therapy with gentle range of motion, local corticosteroid injections, and manipulative therapy. Surgical release is performed as a final option. ${ }^{6,10,15,17,19,25}$ Surgical treatment or operative manipulation for the stiff and painful shoulder is indicated only after a lengthy period of nonoperative therapy has failed. ${ }^{6,10-12,17}$

Arthroscopic intervention in the treatment of the primary frozen shoulder or the secondary stiff and painful shoulder has recently been popularized. $1,6,7,15,17,18,23,24$ It is thought that both the functional and economic loss associated with a long nonoperative rehabilitation course can be greatly reduced by a minimally invasive arthroscopic capsular release..$^{1,5-8,11,24}$ The indications, appropriate timing, suitable candidates, and long-term outcomes for the arthroscopic treatment of the stiff and painful shoulder are presently being evaluated and defined. $1,6,7,11,15,17,18,23,24$ In addition, arthroscopic intervention for a condition that is generally regarded as self limiting must be shown to have low morbidity and good pain relief and to provide an early return to full activities to justify the operative risk. ${ }^{5,6,8}$ Before establishing a protocol for the refractory stiff and painful shoulder that involves an arthroscopic evaluation and capsular release, the safety of this procedure should be evaluated experimentally in a cadaveric model. The purpose of this study was to evaluate and define the anatomic proximity of several neurovascular structures in relation to the arthroscopically released joint capsule in a cadaveric model. 


\section{MATERIALS AND METHODS}

Seven fresh cadaveric shoulders (average age, 68.3 years; range, 42 to 90 ; three male and four female) underwent intraarticular arthroscopic capsular releases with the aid of electrocautery. Each cadaveric hemisection was placed in the lateral position with the aid of a scapular clamp while the humerus was held in approximately $45^{\circ}$ of abduction and $20^{\circ}$ of flexion using traction applied to the distal humerus.

Each shoulder was distended with $60 \mathrm{ml}$ of water and the arthroscope (a $3.5 \mathrm{~mm}$ scope $/ 30^{\circ}$, Dyonics Corp., Andover, Massachusetts) was inserted into a standard posterior portal. A sequential examination was performed as described by Snyder. ${ }^{21}$ The subacromial space was not evaluated. The anterior portal was made with the aid of a switching stick passed from inside the glenohumeral joint over the border of the subscapularis tendon. Through the anterior portal the arthroscopic electrocautery tip (Instrument Makar Inc., Okemos, Michigan) was introduced into the glenohumeral joint. Using the posterior portal for visualization, the surgeon used the electrocautery on the cutting setting (Level 10, 350 watts) to incise the anterior capsule parallel to the anterior rim of the glenoid labrum, approximately $1 \mathrm{~cm}$ lateral to the glenoid (Fig. 1). When as much capsule as possible was released through the ante-

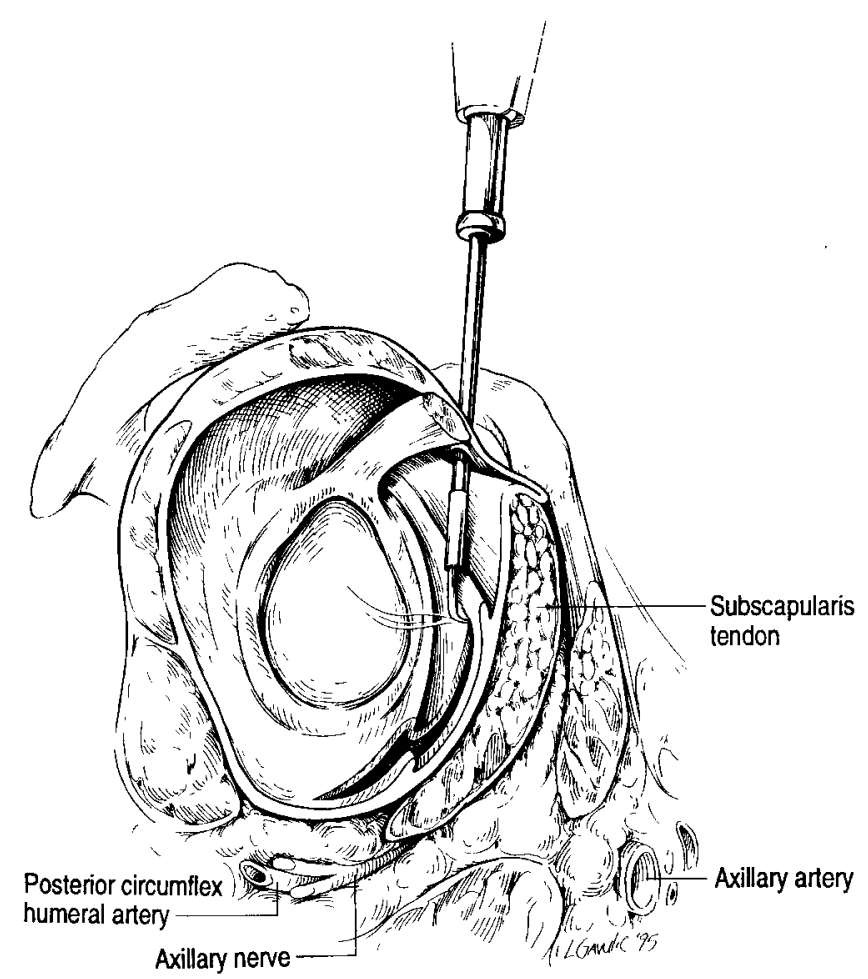

Figure 1. Schematic drawing of the capsular release with the aid of the electrocautery device inserted through the anterior portal. The electrocautery unit has released the anterior band of the inferior glenohumeral ligament and is approaching the middle glenohumeral ligament. Neighboring anatomic structures are labeled. rior portal, the arthroscope was placed anteriorly and the remaining posterior capsule was released with the electrocautery tip in the posterior portal. This ensured that the capsule was released circumferentially under direct observation. Because the capsule blends with the rotator cuff tendons laterally, care was taken to release the medial capsule within $1 \mathrm{~cm}$ of the glenoid labrum, thereby avoiding the rotator cuff tendons anteriorly, superiorly, and posteriorly. The biceps tendon was preserved.

Three parallel $\mathrm{K}$-wires ( 0.0625 inches in diameter) were then placed percutaneously under arthroscopic observation across the glenohumeral joint in the sagittal plane through the released capsule. The arthroscope was then withdrawn and, with the aid of a polystyrene plastic block, the shoulder was secured in the lateral arthroscopic position ( $45^{\circ}$ of abduction, $20^{\circ}$ of flexion). The cadaveric shoulder was then frozen in this position at $-18^{\circ} \mathrm{C}$.

While frozen, the specimen was transected in a sagittal plane just lateral to the released capsule. The parallel $\mathrm{K}$-wires were used as a guide. After sectioning, the Kwires were removed along with the medial fragment of the humeral head. While the glenoid hemisection remained frozen, careful anatomic dissection was performed. The biceps tendon, glenohumeral ligaments, released capsular remnant, rotator cuff tendons and muscles, teres major muscle, long head of the triceps, axillary nerve, posterior circumflex humeral artery, brachial artery, pectoralis major muscle, and latissimus dorsi muscle were identified. Calipers were then used to measure the shortest distance between the released capsular remnant and the axillary nerve, the posterior circumflex humeral artery, and the brachial artery at their nearest points. Each measurement was performed three separate times for each specimen by one author and an average distance was calculated (Table 1 ). These average values were used to determine small-sample confidence intervals (CI) at the 95\% level. All measurements and dissections were performed while the specimens were frozen and maintained in the simulated lateral arthroscopic position. The course of the axillary nerve was traced in every specimen (Fig. 2).

TABLE 1

Average Distance of Major Arteries and Nerves from the Capsular Remnant (in millimeters) ${ }^{a}$

\begin{tabular}{ccccc}
\hline Specimen & Age/Sex & Axillary nerve & $\begin{array}{c}\text { Posterior } \\
\text { circumflex } \\
\text { humeral artery }\end{array}$ & Brachial artery \\
\hline 1 & $72 / \mathrm{M}$ & 9.0 & 9.0 & 30.0 \\
2 & $42 / \mathrm{M}$ & 6.5 & 6.5 & 12.3 \\
3 & $42 / \mathrm{M}$ & 5.7 & 5.7 & 11.0 \\
4 & $90 / \mathrm{F}$ & 4.8 & 6.3 & 16.6 \\
5 & $90 / \mathrm{F}$ & 7.0 & 9.8 & 11.2 \\
6 & $71 / \mathrm{F}$ & 8.8 & 10.5 & 14.0 \\
7 & $71 / \mathrm{F}$ & 7.5 & 9.3 & 16.7 \\
Average & & 7.04 & 8.2 & 15.97 \\
Range & & $4.5-9.5$ & $5.0-11.0$ & $10.0-30.0$ \\
$95 \%$ CI & & $5.62,8.47$ & $6.42,9.98$ & $9.85,22.09$ \\
\hline
\end{tabular}

${ }^{a}$ Three measurements were taken and averaged for each specimen. 

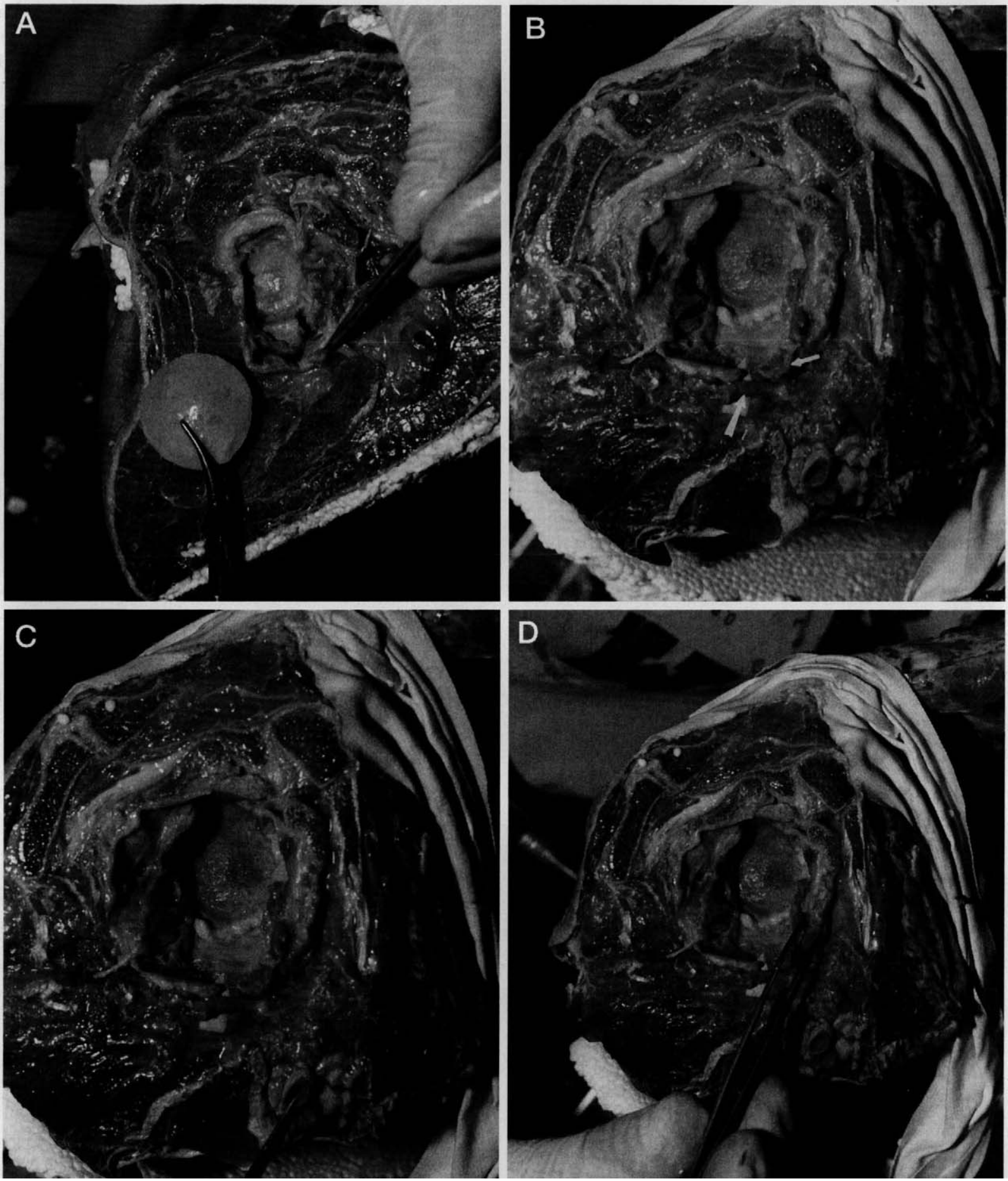

Figure 2. Shoulder hemisections in the sagittal plane from one specimen. The shoulder hemisection was performed with the shoulder frozen in the arthroscopic position ( $45^{\circ}$ of abduction and $20^{\circ}$ of flexion). The hemisection is approximately $1 \mathrm{~cm}$ lateral to the glenoid. A, after sectioning the shoulder in the sagittal plane just lateral to the glenoid, the residual humeral head is easily removed and is held with a hemostat. The axillary nerve is held by the forceps. $B$, the axillary nerve is dissected free and placed on background material for contrast. The tip of the large arrow points to the axillary nerve, and the tip of the small arrow is on the released capsular edge. $C$, the same specimen with the probe on the brachial artery. $D$, as the course of the axillary nerve is traced medially and superiorly, the subscapularis muscle (between forceps) becomes interposed between the axillary nerve and capsular edge. 


\section{RESULTS}

The average distance between the released capsular edge and the axillary nerve was $7.04 \mathrm{~mm}(95 \% \mathrm{CI}, 5.62,8.47)$. The average distance between the posterior circumflex humeral artery and the released capsular edge was 8.2 $\mathrm{mm}(95 \% \mathrm{CI}, 6.41,9.99)$, and the distance between the capsule and brachial artery averaged $15.97 \mathrm{~mm}$ (95\% CI, 9.85, 22.09) (Table 1).

No specimens demonstrated damage to the rotator cuff tendons immediately adjacent to the released capsule. In every specimen the axillary nerve was found to lie anterior to the inferior edge of the subscapularis muscle at the level of the glenoid rim. The nerve continued laterally and inferiorly, traveling under the subscapularis muscle and close to the capsule approximately $1.7 \mathrm{~cm}$ lateral to the inferior glenoid rim.

\section{DISCUSSION}

The primary stiff and painful shoulder has been described in 1872 by Duplay ${ }^{4}$ as a "painful and stiffening shoulder," in 1934 by Codman ${ }^{3}$ as the "frozen shoulder," and in 1945 by Neviaser ${ }^{13}$ as "adhesive capsulitis" based on the finding at arthrography of the shoulder joint. The causes and treatment of this condition continue to frustrate physicians. ${ }^{5,6,11,12}$ If untreated, the joint will eventually recover motion over a period of 12 to 42 months, with a mean recovery period of near 30 months. ${ }^{6,11,16,22}$ Recently, several authors have included arthroscopic procedures in the treatment of the stiff and painful shoulder. ${ }^{1,6,7,15,17,18,20,23,24}$ Arthroscopic procedures not only provide diagnostic information, they also eliminate the need for postoperative immobilization or hospitalization. ${ }^{1,6,7,11,15,17,18,20,23,24}$ Furthermore, arthroscopic surgery allows for capsular distention, debridement of intraarticular adhesions, and the release of contracted capsular structures before or after manipulations of the extremity. ${ }^{1,6,7,15,17,18,20,23,24}$ Moreover, additional pathologic conditions such as degenerative acromioclavicular joints, subacromial impingement, or partial rotator cuff tears can be addressed concurrently. ${ }^{17,18}$

Pollock et al. ${ }^{17}$ used manipulation under anesthesia before the insertion of the arthroscope. After manipulation, they carefully debrided the contracted capsular structures while avoiding debridement inferiorly because the axillary nerve was thought to be at risk secondary to the inferior capsular tear that occurred from the manipulation. Using this technique, they reported an $83 \%$ satisfaction rate in patients without diabetes and a $64 \%$ satisfaction rate in patients with diabetes after 31 months. Capsular releases before manipulation or in those patients who had failed results from a previous manipulation have been performed by Warner et al., ${ }^{23}$ who released the capsule arthroscopically with the aid of electrocautery. They reported low morbidity with only one case of early, isolated shoulder dislocation postoperatively after a 2-year followup.

More recently, Ogilvie-Harris et al. ${ }^{15}$ prospectively divided 40 patients with resistant, stiff, and painful shoul- ders into two groups: one group underwent a manipulation followed by arthroscopy and in the second group the contracted structures were divided using a debrider through arthroscopic means before manipulation. Both groups did equally well with regard to restoration of range of movement after at least 2 years of followup; however, the patients who received arthroscopic division of the contracted structures before manipulation had significantly better pain relief and restoration of function. Segmüller et al. ${ }^{20}$ likewise reviewed the results of 26 shoulders that underwent arthroscopic release of the inferior capsule with a diathermic knife followed by gentle manipulation after failed results with at least 6 weeks of nonoperative therapy. At 13.5 months, $88 \%$ of the patients were very satisfied and had no significant complications. Importantly, two of the three failed results in their series were seen in diabetic patients, and the authors appropriately urged others to use caution when treating patients with both conditions. Manipulation after an arthroscopic release may prove more beneficial than routine manipulations, especially with regard to pain relief. ${ }^{15,20}$

The proximity of several neurovascular structures in the shoulder has been appreciated by several authors. ${ }^{2,6,7,9,12,14,17,23}$ Harryman et al., ${ }^{6,7}$ like Warner et al. ${ }^{23,24}$ released the thickened capsular structures arthroscopically before manipulating the shoulder. In a series of 27 patients, Harryman et al. ${ }^{7}$ noted significant gains in pain relief, motion, and function in short-term followup. The only complication noted was a transient axillary nerve palsy.

Before developing an arthroscopic protocol to address the chronic stiff and painful shoulder, we thought a simple anatomic study to identify the exact proximity of neighboring neurovascular structures to the joint capsule was justified. Bryan et al. ${ }^{2}$ and Loomer and Graham ${ }^{9}$ have specifically evaluated the axillary nerve and its relationship to the anterolateral shoulder capsule and rotator cuff during an open anteroinferior capsular shift for multidirectional instability. Loomer and Graham recommended direct visualization of the axillary nerve before cutting either the subscapularis tendon or the inferior capsule. External rotation was also emphasized so as to relax the tension on the main trunk of the axillary nerve.

Our results show that, with the shoulder in the lateral arthroscopic position ( $45^{\circ}$ of abduction and $20^{\circ}$ of flexion), an average distance of $7.04 \mathrm{~mm}$ separates the axillary nerve and the released capsule $1 \mathrm{~cm}$ lateral to the glenoid labrum. The posterior circumflex humeral artery was 8.2 $\mathrm{mm}$ and the brachial artery was $15.97 \mathrm{~mm}$ from the released capsule. As the axillary nerve is traced medially toward the glenoid, the inferior border of the subscapularis muscle intercedes between the anterior capsule and the axillary nerve. Bryan et al. ${ }^{2}$ found that an average of $3.2 \mathrm{~mm}$ (range, 0.0 to 8.0 ) separated the axillary nerve and the inferior border of the subscapularis tendon, thus emphasizing the need to stay within $1 \mathrm{~cm}$ of the glenoid rim.

The distance from the glenoid rim and the depth of the capsular release can be estimated by knowing the size of the electrocautery tip and using it as a guide. By staying within $1 \mathrm{~cm}$ of the glenoid rim and avoiding penetrating 
the capsule deeper than $4.5 \mathrm{~mm}$ (the distance from the bend to the tip of the electrocautery device), a capsular release can be adequately and safely performed. It should be noted that this study evaluated normal shoulders in the lateral arthroscopic position. The effect of the beach-chair position (arm adducted to the body, no forward elevation, and near $90^{\circ}$ of internal rotation) was not evaluated, and this position may change the relationship of the axillary nerve to the capsule. The exact role of arthroscopic intervention in the treatment of the stiff and painful shoulder remains to be defined; however, the low morbidity seen in early reports and the economic burden of a long protracted recovery make this approach both safe and appealing.

\section{REFERENCES}

1. Andersen $\mathrm{NH}$, Johannsen HV, Sneppen OJ, et al: Frozen shouider: Arthroscopy and manipulation in general anesthesia, followed by early passive mobilization. Ugeskr Laeger 158: 147-150, 1996

2. Bryan WJ, Schauder K, Tullos HS: The axillary nerve and its relationship to common sports medicine shoulder procedures. Am J Sports Med 14: 113-116, 1986

3. Codman EA: The Shoulder. Boston, Thomas Todd Co., 1934

4. Duplay ES: De la Peri-arthrite Scapulo Humerale et des Raideurs de L'epaule Qui en son la Consequence. Arch Gen Med 20: 5131-5142, 1872

5. Ekelund $\mathrm{AL}, \mathrm{Rydell} \mathrm{N}$ : Combination treatment for adhesive capsulitis of the shoulder. Clin Orthop 282: 105-109, 1992

6. Harryman DT: Shoulders: Frozen and stiff. Instr Course Lect 42:247-257, 1993

7. Harryman DT II, Matsen FA III, Sidles JA: Arthroscopic management of refractory shoulder stiffness. J Shoulder Elbow Surg 5: S112, 1996

8. Hulstyn MJ, Weiss APC: Adhesive capsulitis of the shoulder. Orthop Rev 22: 425-433, 1993

9. Loomer R, Graham B: Anatomy of the axillary nerve and its relation to inferior capsular shift. Clin Orthop 243: 100-105, 1989
10. Loyd JA, Loyd HM: Adhesive capsulitis of the shoulder: Arthrographic diagnosis and treatment. Southern Med J 76:879-883, 1983

11. Murnaghan JP: Adhesive capsulitis of the shoulder: Current concepts and treatment. Orthopedics 11: 153-158, 1988

12. Neviaser JS: Adhesive capsulitis and the stiff and painful shoulder. Orthop Clin North Am 11: 327-331, 1980

13. Neviaser JS: Adhesive capsulitis of the shoulder. A study of the pathological findings in periarthritis of the shoulder. I Bone Joint Surg 27: 211-222, 1945

14. Neviaser TJ: Adhesive capsulitis. Orthop Clin North Am 18: 439-443, 1987

15. Ogilvie-Harris DJ, Biggs DJ, Fitsialos DP, et al: The resistant frozen shoulder. Manipulation versus arthroscopic release. Clin Orthop 319: 238-248, 1995

16. Ozaki J, Nakagawa $Y$, Sakurai $G$, et al: Recalcitrant chronic adhesive capsulitis of the shoulder. Role of contracture of the coracohumeral ligament and rotator interval in pathogenesis and treatment. $J$ Bone Joint Surg 71A: 1511-1515, 1989

17. Pollock RG, Duralde XA, Flatow EL, et al: The use of arthroscopy in the treatment of resistant frozen shoulder. Clin Orthop 304: 30-36, 1994

18. Rhee YG, Eum CM: Arthroscopic release in the frozen shoulder. J Shoulder Elbow Surg 5: S26, 1996

19. Rizk TE, Pinals RS, Talaiver AS: Corticosteroid injections in adhesive capsulitis: Investigation of their value and site. Arch Phys Med Rehabil 72: 20-22, 1991

20. Segmüller HE, Taylor DE, Hogan CS, et al: Arthroscopic treatment of adhesive capsulitis. J Shoulder Elbow Surg 4: 403-408, 1995

21. Snyder SJ: Diagnostic arthroscopy of the shoulder: Normal anatomy and variations, in Shoulder Arthroscopy. New York, McGraw-Hill Book Co., 1994, pp 23-41

22. Waldburger $M$, Meier $\mathrm{JL}$, Gobelet $\mathrm{C}$ : The frozen shoulder: Diagnosis and treatment. Prospective study of 50 cases of adhesive capsulitis. Clin Rheumatol 11: 364-368, 1992

23. Warner JJP, Allen A, Marks $P$, et al: Arthroscopic release of chronic, refractory capsular contracture of the shoulder. J Shoulder Elbow Surg 5 : S7, 1996

24. Warner JJP, Allen AA, Marks $P$, et al: Arthroscopic release of refractory capsular contracture of the shoulder. Surgical Demonstration Video, American Academy of Orthopaedic Surgeons, Rosemont, IL, 1995, PC\#25140

25. Williams NE, Seifert MH, Cuddigan JHP, et al: Treatment of capsulitis of the shoulder. Rheumatol Rohabil 14: 236, 1975 